\title{
Total Synthesis of the brevicidine and laterocidine family of lipopeptide antibiotics
}

\author{
Karol Al Ayed, ${ }^{a, \ddagger}$ Ross Ballantine, ${ }^{b, \ddagger}$ Zheng Zhong, ${ }^{\mathrm{c}}$ Yong-Xin Li, ${ }^{\mathrm{c}}$ \\ Stephen A. Cochrane ${ }^{b_{*} *}$ Nathaniel I. Martin ${ }^{a} *$
}

${ }^{a}$ Biological Chemistry Group, Institute of Biology Leiden, Leiden University, Sylviusweg 72, 2333 BE Leiden, The Netherlands.

${ }^{b}$ School of Chemistry and Chemical Engineering, Queen's University Belfast, David Keir Building, Stranmillis Road, Belfast, BT9 5AG, UK.

${ }^{c}$ Department of Chemistry, The University of Hong Kong, Pokfulam Road, Hong Kong, China

These authors contributed equally.

*Corresponding authors email: s.cochrane@qub.ac.uk,n.i.martin@biology.leidenuniv.nl

\begin{abstract}
Brevicidine and laterocidine are two recently discovered lipopeptide antibiotics with promising antibacterial activity. Possessing a macrocyclic core, multiple positive charges, and a lipidated $N$ terminus, these lipopeptides exhibit potent and selective activity against Gram-negative pathogens including polymyxin-resistant isolates. Given the low amounts of brevicidine and laterocidine accessible by fermentation of the producing microorganisms, synthetic routes to these lipopeptides present an attractive alternative. We here report the convenient solid-phase syntheses of both brevicidine and laterocidine and confirm their potent anti-Gram-negative activities.
\end{abstract}

\section{Introduction}

The accelerated appearance of multi-drug resistant bacterial pathogens has led to the worrying speculation that society may soon face a "post-antibiotic" era. ${ }^{1-3}$ The gravity of the antimicrobial resistance (AMR) crisis is most clearly reflected by the spread of the "ESKAPE" pathogens ( $E$. faecium, S. aureus, K. pneumoniae, A. baumannii, P. aeruginosa, and Enterobacter species) a 
group of organisms that are increasingly difficult or impossible to treat with conventional antibiotics. Globally, deaths due to infections with drug-resistant bacteria are nearing one million per year. ${ }^{4}$ Even more worrying are recent projections suggesting that by the year 2050 the number of AMR associated deaths will grow to a staggering ten million per year. ${ }^{4}$

To prioritize the greatest threats currently associated with AMR, the World Health Organization (WHO) recently published its list of priority pathogens. ${ }^{5}$ Among these pathogens, it is exclusively the Gram-negative members of the ESKAPE family that are labeled as "critical", the highest threat level on the WHO list. This is due to the rapidly accelerating rise in antibiotic resistance among Acinetobacter, Pseudomonas, and various Enterobacteriaceae (including Klebsiella and E. coli) causing severe and often deadly bloodstream and pulmonary infections. The AMR threat underscores the importance of pursuing new strategies in discovering and developing the antibiotics of the future.

Using a biosynthetic gene cluster mining strategy, Li and co-workers recently reported the discovery of a promising new class of macrocyclic lipopeptides termed the brevicidines and laterocidines (Fig. 1). ${ }^{6}$ Produced by strains of Brevibacillus laterosporus, brevicidine and laterocidine specifically kill all Gram-negative members of the ESKAPE family, including drugresistant strains. The antibacterial activity of these lipopeptides is promising with MIC values comparable to the polymyxins, the only class of lipopeptides presently used in the clinical treatment of serious Gram-negative infections. Of particular note is the finding that brevicidine and laterocidine effectively kill pathogenic strains featuring the recently reported MCR-type polymyxin resistance mechanism. ${ }^{6}$ Furthermore, the initial report describing the discovery of brevicidine and laterocidine also indicates that these lipopeptide antibiotics show little propensity to induce resistance and have low toxicity towards mammalian cells. ${ }^{6}$

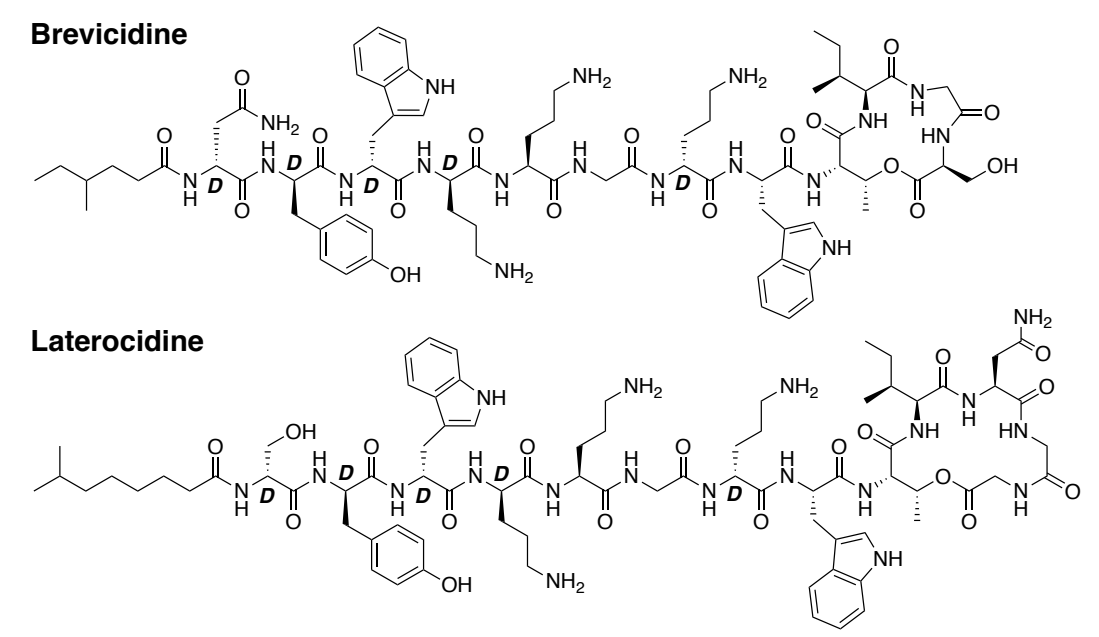

Figure 1. Structures of brevicidine and laterocidine. 
While brevicidine and laterocidine are promising new anti-Gram-negative antibiotics, both compounds are difficult to isolate in significant quantities from natural sources, presenting a major obstacle to investigating their full potential. Brevicidine and laterocidine can be obtained by fermentation of the producing microorganisms, however this labour-intensive process provides limited amounts of material (sub-milligram-per-litre yields in the case of laterocidine). ${ }^{6}$ For this reason, reliable synthetic routes to these lipopeptides present an attractive alternative as a means of providing larger amounts of material for both clinical evaluation and mechanistic studies. Herein we report the first total syntheses of brevicidine and laterocidine, demonstrating that the synthetic compounds have identical ${ }^{1} \mathrm{H}-\mathrm{NMR}$ spectra, RP-HPLC retention times, and antibacterial activities compared to their respective natural products.

\section{Results and Discussion}

Brevicidine and laterocidine share a number of structural features including a C-terminal esterlinked macrocycle of 4- or 5- amino acids, a number of conserved residues including three positively charged ornithines, and a lipidated N-terminus. It was previously demonstrated that the macrocycle is necessary for antibacterial activity of these peptides. ${ }^{6}$ Key to the syntheses of both brevicidine and laterocidine was therefore development of a reliable approach for the introduction of the macrocycle formed via an ester linkage between the C-terminal carboxylate and the corresponding threonine side chain hydroxyl group. For both brevicidine and laterocidine, convenient solid phase approaches were developed that allowed on-resin formation of the key macrocycle and installation of all other amino acids.

\section{Synthesis of Brevicidine}

In approaching the synthesis of brevicidine we initially investigated a strategy starting from Gly11 loaded on 2-chlorotrityl chloride (CTC) to generate a linear peptide that would subsequently be cyclized in solution. To do so the required Thr9-Ser12 linkage was envisioned to be installed as a preformed, ester-linked dipeptide (Scheme 1). However, while incorporation of the Thr9-Ser12 unit was achieved, further elongation of the peptide failed due to an $O \rightarrow N$ acyl shift that occurred upon removal of the Thr9 Fmoc group (see supporting Scheme S1). As an alternative, we next examined formation of the macrocycle at an earlier stage to assess whether the ester linkage might be more stable when contained in the more conformationally restricted ring system. To implement this approach, Fmoc-Ser-OAll was loaded on to CTC resin via its free side-chain hydroxyl group. Notably, the initial conditions used $(1 \mathrm{~h}, \mathrm{RT})$ resulted in a lower loading than required $(0.07$ $\mathrm{mmol} / \mathrm{g}$ ), so the reaction time was extended to $24 \mathrm{~h}$ along with heating at $45^{\circ} \mathrm{C}$, resulting in an 


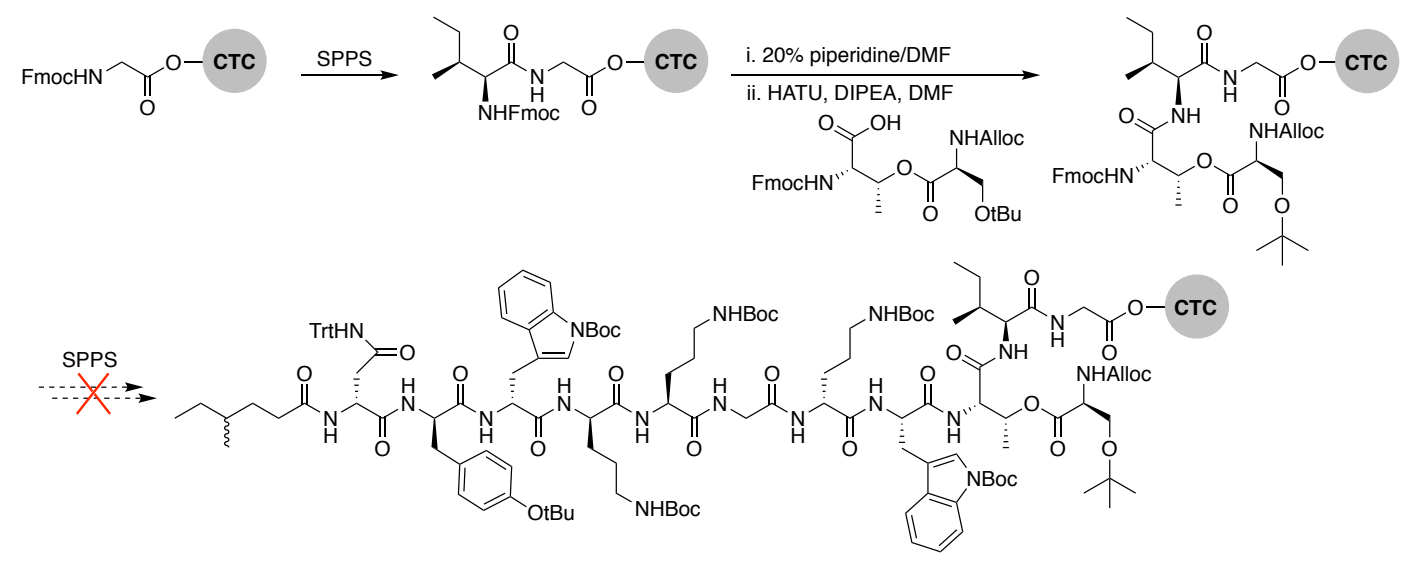

Scheme 1. Initial approach investigated in the synthesis of brevicidine.

improved loading of $0.13 \mathrm{mmol} / \mathrm{g}$. Resin-bound Fmoc-Ser-OAll was then extended to the tetrapeptide using standard Fmoc-SPPS (Scheme 2). At this stage the C-terminal allyl ester was cleanly removed on resin using $\mathrm{Pd}\left(\mathrm{PPh}_{3}\right)_{4} / \mathrm{PhSiH}_{3}$ in $\mathrm{CH}_{2} \mathrm{Cl}_{2}$. Subsequent closure of the macrolactone was then investigated using different coupling conditions. Cyclization of the on-resin tetrapeptide proved to be refractory to both the use of 1-ethyl-3-(3-dimethylaminopropyl) carbodiimide (EDC) and diisopropylcarbodiimide (DIC), with only starting material being obtained after $48 \mathrm{~h}$. Brimble et al. encountered a similar problem in their synthesis of xenetamide, an antimicrobial cyclodepsipeptide isolated from Xenorhabdus nematophilus. ${ }^{7}$ To circumvent the lack of reactivity, the authors successfully employed a modified Yamaguchi esterification, utilizing benzoyl chloride as the coupling reagent. Following this work, we applied similar conditions ( $24 \mathrm{~h}$, RT) to obtain a small amount of the desired cyclic product. Increasing the reaction temperature to $60^{\circ} \mathrm{C}$ resulted in near complete conversion to the cyclic product. Building from the successful formation of the resin-bound tetrapeptide macrolactone, all that remained was to extend the peptide from the $N$-terminal threonine residue with SPPS. It was thought best to proceed with caution at the initial Fmoc deprotection to avoid unwanted $O \rightarrow N$ acyl migration. Therefore, less aggressive Fmoc deprotection conditions (10\% piperidine in DMF) were used in the first deprotection. Gratifyingly, we did not detect any $O \rightarrow N$ acyl migration, even using standard Fmoc deprotection conditions, validating our hypothesis that preforming the C-terminal macrocycle would overcome this issue. The remainder of the peptide was constructed without incident along with coupling of the Nterminal 4-methylhexanoic acid. Following global deprotection and resin cleavage and purification by RP-HPLC, synthetic brevicidine was obtained in an excellent overall yield of $9 \%$ over 28 steps. 


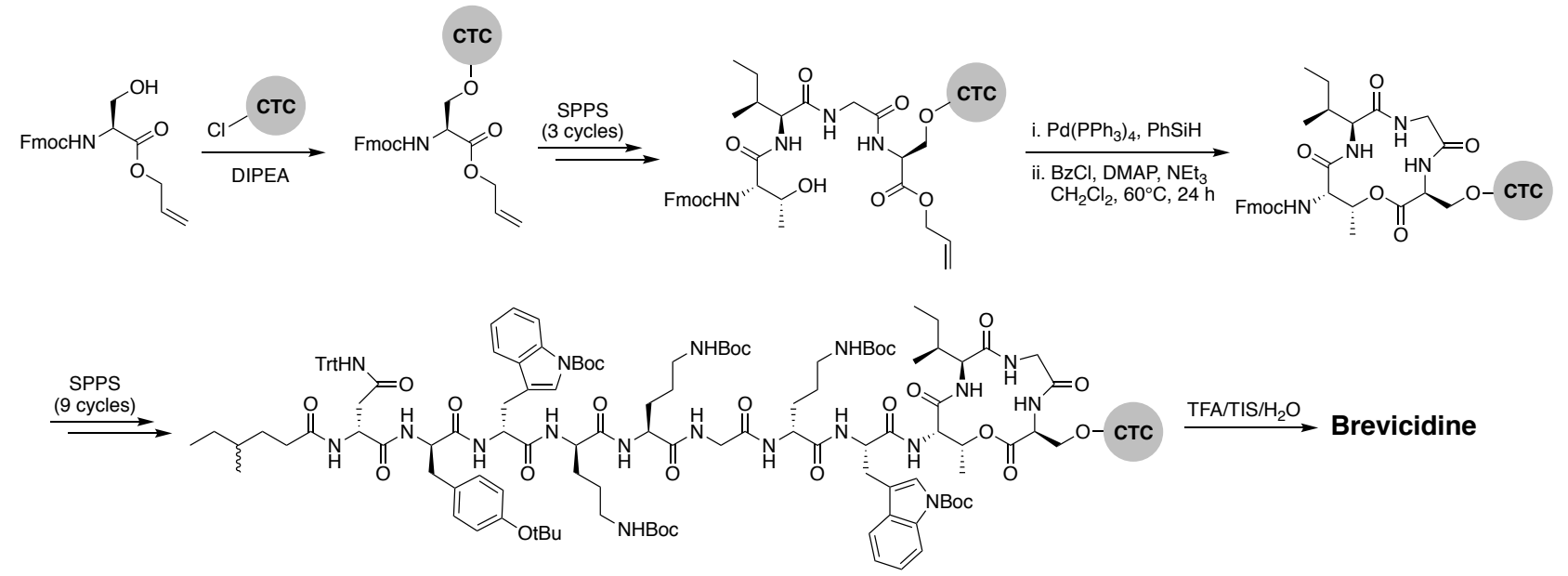

Scheme 2. Synthesis of brevicidine.

\section{Synthesis of Laterocidine}

The synthetic strategy initially pursued for the preparation of laterocidine was inspired by the successful route developed for brevicidine. To begin, Fmoc-Asp-OAll was loaded onto Rink amide resin via its free side chain carboxylate. Following allyl ester removal, an allyl ester protected GlyGly dipeptide was next coupled (Scheme 3). The peptide was then built out to the Trp8 residue (so as to avoid possible $O \rightarrow N$ acyl migration later on in the synthesis) with Thr9 successfully installed without side chain protection. Following allyl ester deprotection, formation of the macrolactone was attempted using a variety of conditions, including the modified Yamaguchi esterification, but all failed to provide the desired product in appreciable yield. We ascribe this difficulty in ester formation to the larger five-amino-acid macrocycle found in laterocidine versus the four-amino-acid ring found in brevicidine.

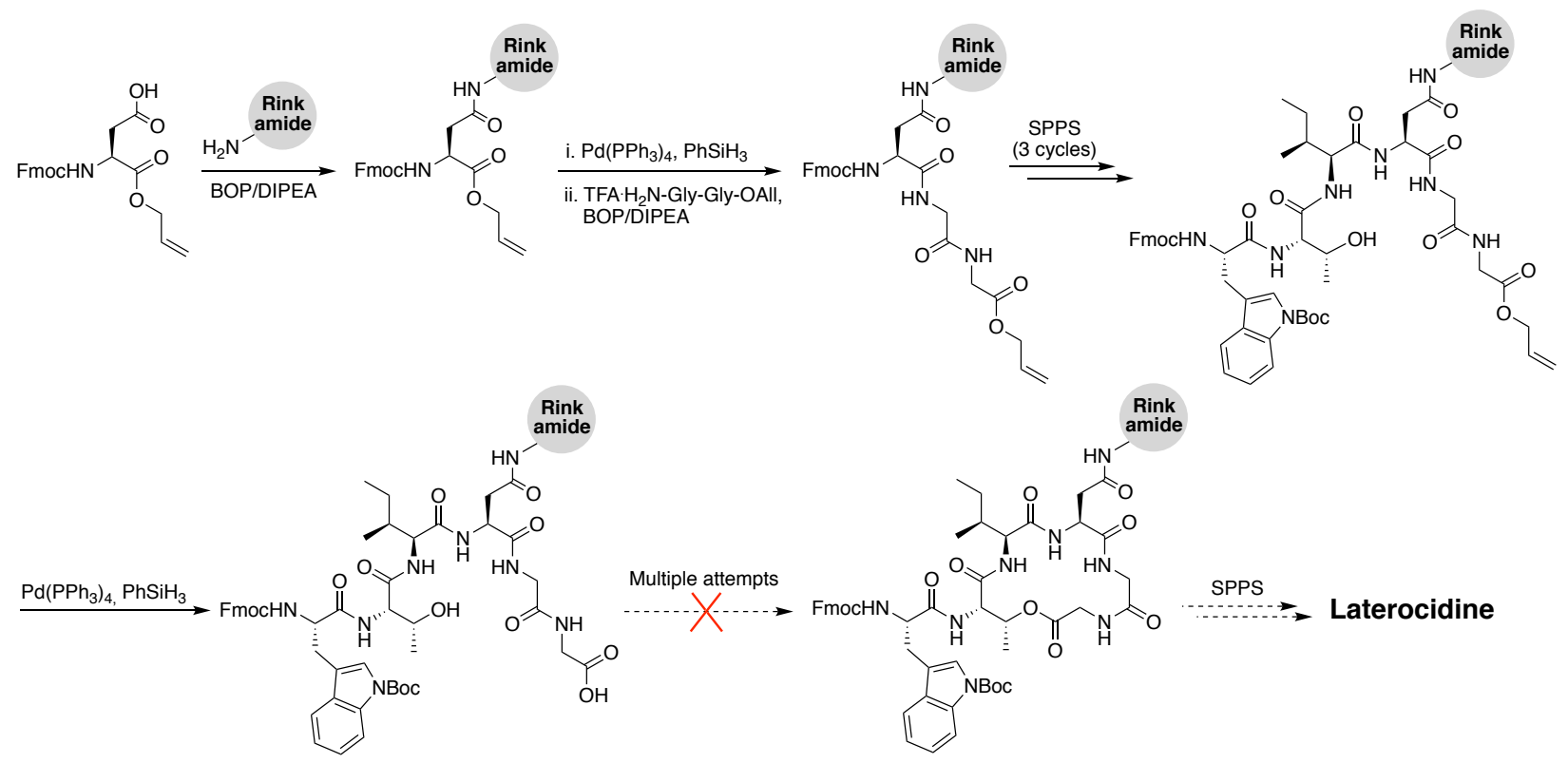

Scheme 3. Initial approach attempted in synthesis of laterocidine. 
As an alternative, we next investigated the possibility of closing the macrocycle via amide bond formation between Gly12 and Gly13 (Scheme 4). To do so, the same Fmoc-Asp-OAll loaded Rink amide resin used in the initial attempt was again employed. Following allyl ester cleavage, an allyl ester protected Gly was coupled after which the peptide was built out up to $\operatorname{Trp} 8$ as before. The ester linkage between the free Thr9 side chain hydroxyl and the Gly13 C-terminal carboxylate was successfully introduced by coupling Alloc-Gly-OH using an on-resin Steglich esterification approach inspired by the Albericio group's synthesis of pipecolidepsin A. ${ }^{8}$ Following removal of the allyl and alloc protecting groups, a BOP/DIPEA mediated macrolactamization resulted in the clean formation of the macrocyclic product. From there the peptide was completed using standard Fmoc-SPPS conditions including N-terminal lipidation with isopelargonic acid. Following cleavage from the resin and global deprotection, the crude lipopeptide was subsequently purified using RPHPLC, yielding laterocidine in $2 \%$ overall yield over 29 steps.

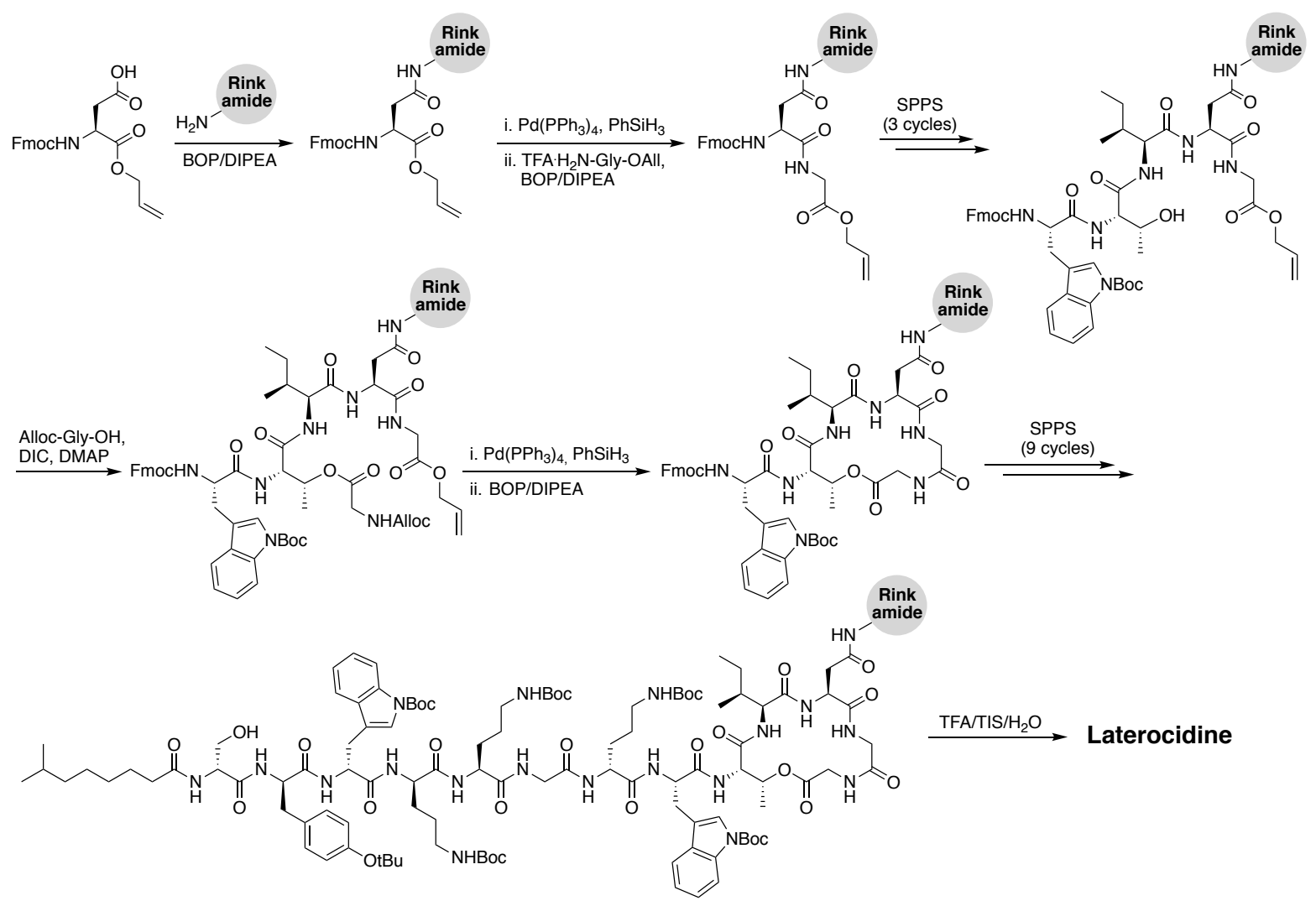

Scheme 4. Successful synthesis of laterocidine.

To confirm the equivalency of the synthetic brevicidine and laterocidine with the natural lipopeptides, the ${ }^{1} \mathrm{H}$ NMR spectra of the synthetic compounds were compared with published data for the natural products revealing them to be indistinguishable (Fig. 2). In addition, RP-HPLC 
analysis of the synthetic lipopeptides and comparison to the natural products, further verified their identity (Supplemental Fig. S1).
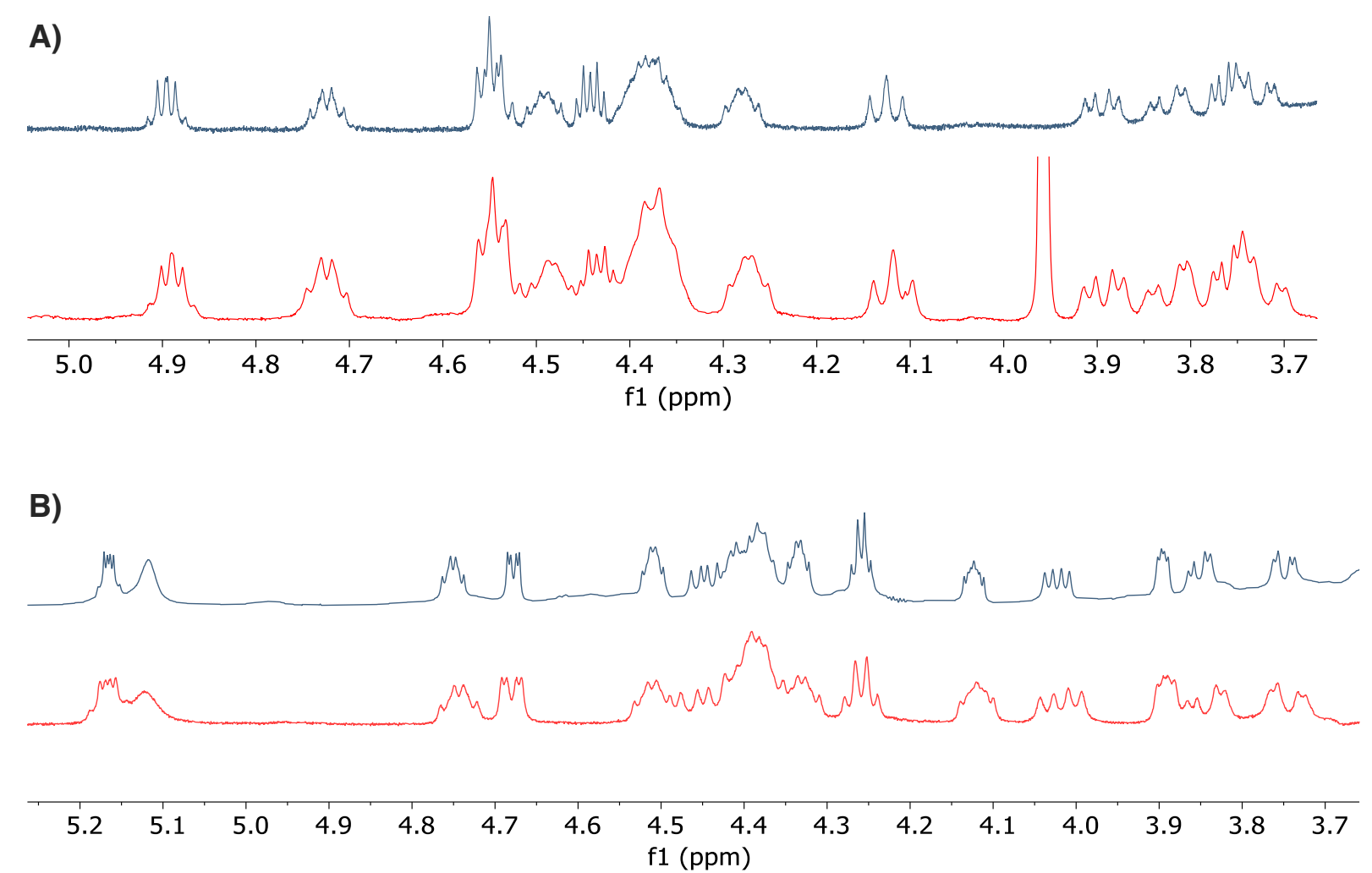

Figure 2. Overlaid portions of ${ }^{l} H$ NMR spectra obtained for synthetic lipopeptides (blue traces) and previously published spectra (red traces) corresponding to: $\boldsymbol{A}$ ) brevicidine and $\boldsymbol{B}$ ) laterocidine. The peak at ca. $3.96 \mathrm{ppm}$ in the published spectrum of brevicidine is attributed to an impurity not present in the synthetic material. Spectra recorded in DMSO-d $d_{6}$ at room temperature. Full ${ }^{1} H$ NMR spectra provided in the supporting information.

\section{Antibacterial Activity}

The antibacterial activities of synthetic brevicidine and laterocidine were next assessed against a panel of Gram-negative pathogens including drug-resistant isolates. In keeping with the previously reported activities of the natural products, the synthetic brevicidine and laterocidine both demonstrated potent activities with MICs on par with the clinically used colistin (Table 1). Also of note is the finding that both brevicidine and laterocidine maintain their activity against strains containing the $m c r$ genotype conferring polymyxin resistance. In recent years concerns have grown over the prevalence of $m c r$-positive strains and the threat they pose to rendering polymyxin treatment ineffective and in so doing breaching one of the last lines of defense available for treating serious Gram-negative infections. ${ }^{9}, 10$ The finding that the activities of brevicidine and laterocidine are not hampered against $m c r$-type strains indicates that the clinical potential of these lipopeptides warrants further investigation. 
Table 1. MIC values for synthetic brevicidine and laterocidine compared with the clinically used lipopeptide antibiotic colistin ${ }^{a}$

\begin{tabular}{lccc}
\hline & Brevicidine & Laterocicine & Colistin \\
\hline E. coli ATCC 25922 & $1-2$ & 1 & $0.25-0.5$ \\
E. coli ATCC 25922 MCR-1 & 2 & 2 & $2-4$ \\
E. coli MCR-1 & $1-2$ & 0.5 & $2-4$ \\
E. coli EQAS MCR-2 & 2 & 1 & $2-4$ \\
K. pneumoniae ATCC 11228 & $1-2$ & 2 & $\leq 0.5$ \\
K. pneumoniae ATCC 13883 & 1 & 1 & 1 \\
K. pneumonia 2048 & $1-2$ & 2 & 2 \\
K. pneumonia JS-123 & 1 & 0.5 & $\leq 0.5$ \\
A. baumannii ATCC 17961 & $2-4$ & 0.5 & $\leq 0.5$ \\
A. baumannii ATCC 17978 & 4 & 0.5 & $\leq 0.5$ \\
A. baumannii 2018-006 & 4 & 2 & $\leq 0.5$ \\
A. baumannii MDR & 4 & 1 & $\leq 0.5$ \\
P. aeruginosa ATCC 27853 & $\leq 0.5$ & $\leq 0.5$ & 1 \\
P. aeruginosa PAO1 & 2 & 1 & $\leq 0.5$ \\
P. aeruginosa NRZ-03961 & 2 & $1-2$ & $\leq 0.5$ \\
P. aeruginosa M-120 & $2-4$ & 1 & 1 \\
S. aureus USA300 & 64 & 64 & $>64$ \\
\hline
\end{tabular}

${ }^{a}$ Minimum inhibitory concentrations reported in $\mu \mathrm{g} / \mathrm{mL}$.

\section{Conclusion}

The first total syntheses of the antimicrobial lipodepsipeptides brevicidine and laterocidine were successfully completed. Brevicidine was obtained in 28 steps from 2-chlorotrityl resin in an overall yield of 9\%, and laterocidine was obtained in 29 steps from Rink-amide resin in an overall yield of $2 \%$. In both syntheses, formation of the macrocycle on-resin at an early stage was found to be most effective, serving to limit deleterious $O \rightarrow N$ acyl shifts at the ester linkage on Thr9, and provided the desired peptides as the major products after cleavage from resin allowing for facile HPLC purification. Divergent strategies were used to form these respective macrocycles. In the case of brevicidine, the four-amino-acid ring was most effectively introduced by means of a modified Yamaguchi esterification between the C-terminal carboxylate of Gly12 and the side chain hydroxyl group of Thr9. For laterocidine, the five-amino-acid ring was formed by first installing the ester linkage by coupling Alloc-Gly-OH to the Thr9 side chain followed by selective Allyl/Alloc deprotection and BOP-mediated macrolactamization between Gly12 and Gly13. Overall, both synthetic strategies are highly robust, yielding brevicidine and laterocidine in quantities that compare well with those obtained by isolation of the natural products from fermentation of the producing organisms. Synthetic brevicidine and laterocidine were shown to have identical ${ }^{1} \mathrm{H}-\mathrm{NMR}$ 
spectra and RP-HPLC elution profiles compared to their natural counterparts confirming the previously reported structures. The antibacterial activities of synthetic brevicidine and laterocidine were also assessed against a panel of Gram-negative pathogens demonstrating their potent antibacterial effect.

In light of the increasing occurrence of Gram-negative pathogens with resistance to conventional antibacterial therapies, the brevicidine and laterocidine family of lipopeptide antibiotics represent promising leads for further development. In addition, the recent discovery of the relacidines, ${ }^{11}$ which show high structural similarity to laterocidine, indicate that these lipopeptide antibiotics may be widespread in nature. Our methodology offers an efficient alternative to isolating these promising natural products from bacterial fermentation and in doing so provides access to quantities of material suitable for further preclinical evaluation and mechanistic studies.

\section{Experimental Details}

\section{Reagents and general methods}

All reagents employed were of American Chemical Society (ACS) grade of higher and were used without further purification unless otherwise stated. Fmoc-Ser-OAll, ${ }^{12}$ Fmoc-Asp-OAll, ${ }^{13}$ $\mathrm{TFA} \cdot \mathrm{NH}_{2}-\mathrm{Gly}-\mathrm{OA} 11,{ }^{14}$ and $\mathrm{TFA} \cdot \mathrm{NH}_{2}-\mathrm{Gly}-\mathrm{Gly}-\mathrm{OA}{ }^{15}{ }^{15}$ were synthesized according to literature procedures. The NMR characterization of brevicidine was obtained using a Bruker Ascend 600 $(600 \mathrm{MHz})$ using DMSO- $d_{6}$. The NMR characterization of laterocidine was obtained on a Bruker AV850 spectrometer $(850 \mathrm{MHz})$ using DMSO- $d_{6}$. LC-MS analyses were performed on a Shimadzu LC-20AD system with a Shimadzu Shim-Pack GISS-HP C18 column $(3.0$ x $150 \mathrm{~mm}, 3 \mu \mathrm{m})$ at $30^{\circ} \mathrm{C}$ and equipped with a UV detector. The following solvent system, at a flow rate of $0.5 \mathrm{~mL} / \mathrm{min}$, was used: solvent A, $0.1 \%$ formic acid in water; solvent B, acetonitrile. Gradient elution was as follows: 95:5 (A/B) for $2 \mathrm{~min}, 95: 5$ to $0: 100(\mathrm{~A} / \mathrm{B})$ over $13 \mathrm{~min}, 0: 100(\mathrm{~A} / \mathrm{B})$ for $2 \mathrm{~min}$, then reversion back to 95:5 (A/B) over $1 \mathrm{~min}, 95: 5(\mathrm{~A} / \mathrm{B})$ for $2 \mathrm{~min}$. This system was connected to a Shimadzu 8040 triple quadrupole mass spectrometer (ESI ionisation).

\section{Preparative HPLC}

Brevicidine was purified using a Perkin Elmer HPLC system composed of a 200 series binary pump, UV/Vis detector monitoring at $220 \mathrm{~nm}$, vacuum degasser, Rheodyne $7725 \mathrm{i}$ injector equipped with a $2 \mathrm{~mL}$ sample loop and Phenomenex Luna C18 column (21.2 x $250 \mathrm{~mm}, 5 \mu \mathrm{m})$. The following solvent system, at a flow rate of $10 \mathrm{~mL} / \mathrm{min}$, was used: solvent A, $0.1 \%$ TFA in water; solvent B, acetonitrile. Gradient elution was as follows: 80:20 (A/B) for $5 \mathrm{~min}, 80: 20$ to 45:55 
(A/B) over $30 \mathrm{~min}, 45: 55$ to 0:95 (A/B) over $3 \mathrm{~min}, 0: 95(\mathrm{~A} / \mathrm{B})$ for $3 \mathrm{~min}$ then reversion back to 80:20 (A/B) over $2 \mathrm{~min}, 80: 20(\mathrm{~A} / \mathrm{B})$ for $5 \mathrm{~min}$.

Laterocidine was purified using a BESTA-Technik system with a Dr. Maisch Reprosil Gold 120 C18 column $(25 \times 250 \mathrm{~mm}, 10 \mu \mathrm{m})$ and equipped with a ECOM Flash UV detector monitoring at $214 \mathrm{~nm}$ and $254 \mathrm{~nm}$. The following solvent system, at a flow rate of $12 \mathrm{~mL} / \mathrm{min}$, was used: solvent A, $0.1 \%$ TFA in water/acetonitrile 95/5; solvent B, $0.1 \%$ TFA in water/acetonitrile 5/95. Gradient elution was as follows: 100:0 (A/B) for $5 \mathrm{~min}, 100: 0$ to $50: 50(\mathrm{~A} / \mathrm{B})$ over $50 \mathrm{~min}, 50: 50$ to $0: 100$ (A/B) for 3min, then reversion back to 100:0 (A/B) over $1 \mathrm{~min}, 100: 0$ (A/B) for $5 \mathrm{~min}$.

\section{Synthesis of Brevicidine}

To a flame dried $25 \mathrm{~mL}$ round bottom flask was added Fmoc-Ser-OAll (110 mg, $0.300 \mathrm{mmol})$ and dry dichloromethane (10.0 mL). 2-Chlorotrityl chloride resin (CTC) (1.00 g, $\left.0.81 \mathrm{mmol} \mathrm{g}^{-1}\right)$ and DIPEA $(210 \mu \mathrm{L}, 1.20 \mathrm{mmol})$ were added. The suspension was stirred under argon for 48 hours, after which the resin was filtered through a manual SPPS vessel and washed with DCM (4 x $5 \mathrm{~mL})$. The resin was then capped by adding a solution of methanol, DIPEA and DCM (3 mL, $10: 5: 85)$ and bubbled with argon for 1 hour. The solution was discharged and the resin was washed with DCM $(3 \times 5 \mathrm{~mL})$ before being dried under a stream of argon, a small portion of resin was then used to ascertain the loading. Estimation of loading level of first residue onto resin was calculated via an Fmoc loading test, as described by Gude et al. ${ }^{16}$

Standard Fmoc SPPS protocol was used to extend the peptide to the linear Fmoc-Thr-Ile-Gly-Ser peptide. Specifically, resin $(0.1 \mathrm{mmol})$ was added to a manual SPPS vessel and bubbled in DMF (3 $\mathrm{mL}$ ) to swell. The solvent was discharged and the resin was bubbled in an Fmoc deprotection solution of $20 \%$ piperidine in DMF ( $3 \times 3 \mathrm{~mL}, 2 \times 1$ min then $1 \times 5 \mathrm{~min})$ with argon. The resin was washed with DMF (3 x $3 \mathrm{~mL}$ ) and a coupling solution of amino acid (6 equiv), HATU (6 equiv) and DIPEA (12 equiv) in DMF (3 mL) was added. The solution was then bubbled with argon for 1 hour, before the solution was discharged and the resin washed with DMF $(3 \times 3 \mathrm{~mL})$. This process was repeated to obtain on-resin linear Fmoc-Thr-Ile-Gly-Ser. A portion of this on-resin allyl protected tetrapeptide (78.0 mg. $0.01 \mathrm{mmol})$ was added to a manual SPPS vessel and bubbled in DCM (3 mL) with argon for 15 minutes. The solvent was discharged and an allyl deprotection solution of tetrakis (triphenylphosphine) palladium (231 mg, $0.200 \mathrm{mmol})$ and phenylsilane (123 $\mu \mathrm{L}, 0.998 \mathrm{mmol})$ in DCM and DMF (1:1, $2 \mathrm{~mL})$ was added. The solution was bubbled with argon for 2 hours in darkness, after which the deprotection solution was discharged and the resin was washed with DMF (3 x $3 \mathrm{~mL}), 0.5 \%$ sodium diethyldithiocarbamate solution in DMF (4 x $5 \mathrm{~mL}), \mathrm{DMF}(4 \times 5 \mathrm{~mL})$ 
and DCM (4 x $5 \mathrm{~mL})$. The resin was dried under argon, then added to a $5 \mathrm{~mL}$ flame dried round bottom flask under argon. Dry dichloromethane $(3 \mathrm{~mL})$ was added and the suspension stirred for 15 minutes. Benzoyl chloride $(13.0 \mu \mathrm{L}, 0.112 \mathrm{mmol})$, triethylamine $(3.00 \mu \mathrm{L}, 22.0 \mu \mathrm{mol})$ and catalytic DMAP ( 1 crystal) were added and the reaction mixture was stirred overnight at $60^{\circ} \mathrm{C}$. The resin was then filtered through a manual SPPS vessel and washed with DMF (3 x $5 \mathrm{~mL})$ and DCM $(3 \times 5 \mathrm{~mL})$ before being dried under argon. A small sample was cleaved using a $2 \%$ TFA solution in DCM (1 $\mathrm{mL}$ ). The cleavage cocktail was gently agitated for 1 hour and filtered through a glass wool plug. The filtrate was concentrated with a gentle stream of argon before being analysed by LC-MS $\left([\mathrm{M}+\mathrm{H}]^{+}\right.$calculated for $\mathrm{C}_{30} \mathrm{H}_{36} \mathrm{~N}_{4} \mathrm{O}_{8}$ 581.2, found (LC-MS) 581.5). Following this modified Yamaguchi esterification, the synthesis of brevicidine was completed using standard Fmoc SPPS protocols as described above after which 4-methylhexanoic acid was coupled to the N-terminus. The dried resin was then added to a cleavage cocktail of TFA, TIPS and distilled water $(10 \mathrm{~mL}, 95$ : $2.5: 2.5$ ) and heated to $37^{\circ} \mathrm{C}$ for 1 hour. The suspension was filtered through a glass wool plug and the filtrate concentrated under vacuum. Diethyl ether was used to precipitate the crude peptide which was then centrifuged and washed with additional diethyl ether. The suspension was centrifuged and the pellet dissolved in a minimal amount of 1:1 acetonitrile and water solution with $0.1 \%$ TFA. The crude mixture was subsequently purified by RP-HPLC as described above. Fractions were assessed by LC-MS and those containing clean product were pooled and lyophilized to yield brevicidine as a white powder. Yield: $13 \mathrm{mg}, 9 \%$ over 28 steps. HPLC retention time 23.3 min; $[\mathrm{M}+3 \mathrm{H}]^{3+}$ calculated for $\mathrm{C}_{74} \mathrm{H}_{106} \mathrm{~N}_{18} \mathrm{O}_{17}$ 507.3, found (LC-MS) 507.8.

\section{Synthesis of Laterocidine}

Rink Amide MBHA resin (2.0 g, $\left.0.67 \mathrm{mmol} \mathrm{g}^{-1}\right)$ was loaded by overnight coupling via the free sidechain carboxylate of Fmoc-Asp-OAll (2.65 g, 6.70 mmol, 2 eq.) with BOP (2.96 g, 6.70 mmol, 2 eq.) and DiPEA (2.33 mL, 13.4 mmol, 4 eq.) in $150 \mathrm{~mL}$ of DMF. After capping with $\mathrm{AcO}_{2}$ : pyridine $(3: 2, \mathrm{v} / \mathrm{v})$ for $30 \mathrm{~min}$ the resin loading was determined to be $0.37 \mathrm{mmol} \mathrm{g}^{-1}$. The loaded resin (680 mg, $0.25 \mathrm{mmol})$ was treated with $\mathrm{Pd}\left(\mathrm{PPh}_{3}\right)_{4}\left(75 \mathrm{mg}, 0.075 \mathrm{mmol}, 0.3\right.$ eq.) and $\mathrm{PhSiH}_{3}$ (0.75 mL, $7.5 \mathrm{mmol}, 30$ eq.) in DCM (ca. $15 \mathrm{~mL}$ ) under nitrogen for 1 hour. The resin was subsequently washed with DCM $(5 \times 10 \mathrm{~mL})$, followed by a solution of diethyldithiocarbamic acid trihydrate sodium salt $\left(5 \mathrm{mg} \mathrm{mL}^{-1}\right.$ in DMF, $\left.5 \times 10 \mathrm{~mL}\right)$, and DMF $(5 \times 10 \mathrm{~mL})$. TFA $\mathrm{H}_{2} \mathrm{~N}-\mathrm{Gly}-$ OAll (115 mg, $0.5 \mathrm{mmol}, 2$ eq.) was then coupled using BOP (221 mg, $0.5 \mathrm{mmol}, 2$ eq.) and DiPEA (174 $\mu \mathrm{L}, 1.0 \mathrm{mmol}, 4$ eq.) under nitrogen flow for 1 hour. The next 3 amino acids (Ile10, Thr9, Trp8) were coupled manually $(1 \mathrm{~h})$ under nitrogen flow via standard Fmoc solid-phase peptide synthesis (SPPS) (resin : Fmoc-AA : BOP : DiPEA, $1: 4: 4: 8$ molar eq.). DMF (5 mL) was used as solvent and Fmoc deprotections ( $2 \mathrm{~min}$ then $10 \mathrm{~min}$ ) were carried out with $5 \mathrm{~mL}$ piperidine : 
DMF (1 : 4, v/v). The following Fmoc amino acids were used: Fmoc-Ile-OH, Fmoc-Thr-OH (used without side chain protection), and Fmoc-Trp(Boc)-OH. After coupling of Fmoc-Trp(Boc)-OH esterification of the Thr side chain was achieved by treating the resin-bound peptide with AllocGly-OH (596 mg, 3.75 mmol, 15 eq.), DIC (0.59 mL, 3.75 mmol, 15 eq. ) and DMAP (15 mg, 0.13 mmol, 0.5 eq. ) in $8 \mathrm{~mL} \mathrm{DCM} \mathrm{:} \mathrm{DMF} \mathrm{(3:1,} \mathrm{v/v)} \mathrm{for} 18 \mathrm{~h}$ under nitrogen. The resin was treated with $\mathrm{Pd}\left(\mathrm{PPh}_{3}\right)_{4}(75 \mathrm{mg}, 0.075 \mathrm{mmol})$ and $\mathrm{PhSiH}_{3}(0.75 \mathrm{~mL}, 7.5 \mathrm{mmol})$ in DCM (ca. $\left.15 \mathrm{~mL}\right)$ under nitrogen for $2 \mathrm{~h}$ before being washed with DCM $(5 \times 10 \mathrm{~mL})$, followed by a solution of diethyldithiocarbamic acid trihydrate sodium salt $\left(5 \mathrm{mg} \mathrm{mL}^{-1}\right.$ in DMF, $\left.5 \times 10 \mathrm{~mL}\right)$, and DMF $(5 \times$ $10 \mathrm{~mL}$ ). The peptide was then cyclized using BOP (442 mg, $1.0 \mathrm{mmol}, 4$ eq. ) and DiPEA (0.35 $\mathrm{mL}, 2.0 \mathrm{mmol}, 8$ eq.) for $2 \mathrm{~h}$ in $5 \mathrm{~mL}$ DMF under nitrogen flow. The remaining linear $\mathrm{N}$-terminal section of the peptide was then synthesized using the standard SPPS protocol mentioned above. The following Fmoc amino acids were used: Fmoc-D-Ser(tBu)-OH, Fmoc-D-Tyr(tBu)-OH, Fmoc-D$\operatorname{Trp}(\mathrm{Boc})-\mathrm{OH}$, Fmoc-D-Orn(Boc)-OH, Fmoc-L-Orn(Boc)-OH, Fmoc-Gly. Following the final Fmoc removal step, isopelargonic acid ( $79 \mathrm{mg}, 0.5 \mathrm{mmol}, 2$ eq.) was coupled using BOP (221 mg, $0.5 \mathrm{mmol}, 2$ eq.) and DiPEA (174 $\mu \mathrm{L}, 1.0 \mathrm{mmol}, 4$ eq.) in $5 \mathrm{~mL}$ of DMF overnight, under nitrogen flow. Final deprotection was carried out by treating the resin with TFA : TIS : H2O (95: $2.5: 2.5$, $10 \mathrm{~mL}$ ) for $90 \mathrm{~min}$. The reaction mixture was filtered through cotton, the filtrate precipitated in MTBE : petroleum ether $(1: 1)$, and the resulting precipitate washed once more with MTBE : petroleum ether $(1: 1)$. The crude cyclic peptide was lyophilized from $\mathrm{tBuOH}: \mathrm{H}_{2} \mathrm{O}(1: 1)$ and purified with reverse phase HPLC. Pure fractions were pooled and lyophilized to yield laterocidine in $>95 \%$ purity as a white powder. Yield: $8 \mathrm{mg}, 2 \%$ over 29 steps. $[\mathrm{M}+\mathrm{H}]^{+}$calculated for, $\mathrm{C}_{78} \mathrm{H}_{113} \mathrm{~N}_{19} \mathrm{O}_{18} 1604.9$ found (LCMS) 1604.7.

\section{MIC determinations}

Minimum inhibitory concentrations (MICs) were determined by broth microdilution according to CLSI guidelines. ${ }^{17}$ Blood agar plates were inoculated with glycerol stocks of E. coli ATCC 25922, E. coli MCR-1 (clinical isolate from Utrecht Medical Centre, NL), E. coli EQAS MCR-2 (clinical isolate from Wageningen University and Research, NL), K. pneumoniae ATCC 11228, $K$. pneumoniae ATCC 13883, K. pneumonia 2048 (clnical isolate from Vrije Universiteit Amsterdam Medical Centre, NL), K. pneumonia JS-123 (clinical isolate from from Utrecht Medical Centre, NL), A. baumannii ATCC 17961, A. baumannii ATCC 17978, A. baumannii 2018-006 (clinical isolate from Rijksinstituut voor Volksgezondheid en Milieu, NL), A. baumannii MDR (clinical isolate from Vrije Universiteit Amsterdam Medical Centre, NL), P. aeruginosa ATCC 27853, P. aeruginosa PAO1, $P$. aeruginosa NRZ-03961 (Reference strain from Das Nationale Referenzzentrum für gramnegative Krankenhauserreger, DE), P. aeruginosa M-120 (clinical isolate 
from Leiden University Medical Centre, NL) and S. aureus USA300 (clinical isolate from Texas Children's Hospitalm, USA). E. coli 25922 MCR1 was grown on LB agar supplemented with kanamycin. The inoculated agar plates were then incubated for 16 hours at $37^{\circ} \mathrm{C}$. Individually grown colonies were subsequently used to inoculate $3 \mathrm{~mL}$ aliquots of TSB that were then incubated at $37^{\circ} \mathrm{C}$ with shaking at $220 \mathrm{rpm}$. In parallel, the lipopeptide antibiotics to be assessed were serially diluted with Mueller-Hinton broth (MHB) in polypropylene 96-well plates (50 $\mu \mathrm{L}$ in each well). Once the $\mathrm{OD}_{600}$ of the bacterial suspensions reached 0.5 , the bacteria were diluted with MHB (final concentration $2 \times 10^{5} \mathrm{CFU} \mathrm{mL}^{-1}$ ) and added to the microplates containing the test compounds (50 $\mu \mathrm{L}$ to each well, final volume: $100 \mu \mathrm{L}$ ). The well-plates were sealed with an adhesive membrane and after 16 hours of incubation at $37^{\circ} \mathrm{C}$ with shaking at $220 \mathrm{rpm}$ the wells were visually inspected for bacterial growth. MIC values were defined as the lowest concentration of the compound that prevented visible growth of bacteria.

\section{Supporting Information}

Supplemental figures: Analytical RP-HPLC traces and 1D and 2D-NMR spectra

\section{Acknowledgements}

We thank Tom Wood for assistance with MIC assays. Financial support provided by the European Research Council (ERC consolidator grant to NIM, grant agreement no. 725523) and the Engineering and Physical Sciences Research Council (EPSRC standard grant to SAC, grant agreement no. EP/T01783X/1).

\section{References}

[1] Bierbaum, G., and Sahl, H. G. (2014) The search for new anti-infective drugs: untapped resources and strategies, Int J Med Microbiol 304, 1-2.

[2] Brown, E. D., and Wright, G. D. (2016) Antibacterial drug discovery in the resistance era, Nature 529, 336-343.

[3] Brown, E. D. (2015) Gram-Negative Resistance, ACS Infect Dis 1, 507.

[4] O'Neill, J. (2014 "Antimicrobial Resistance: Tackling a Crisis for the Health and Wealth of Nations." from http://www.jpiamr.eu/wp-content/uploads/2014/12/AMR-Review-PaperTackling-a-crisis-for-the-health-and-wealth-of-nations_1-2.pdf.) Antimicrobial Resistance: Tackling a Crisis for the Health and Wealth of Nations.

[5] https://www.who.int/news-room/detail/27-02-2017-who-publishes-list-of-bacteria-for-whichnew-antibiotics-are-urgently-needed. 
[6] Li, Y. X., Zhong, Z., Zhang, W. P., and Qian, P. Y. (2018) Discovery of cationic nonribosomal peptides as Gram-negative antibiotics through global genome mining, Nat Commun 9, 3273.

[7] Hung, K. Y., Harris, P. W., Heapy, A. M., and Brimble, M. A. (2011) Synthesis and assignment of stereochemistry of the antibacterial cyclic peptide xenematide, Org Biomol Chem 9, 236-242.

[8] Pelay-Gimeno, M., Garcia-Ramos, Y., Jesus Martin, M., Spengler, J., Molina-Guijarro, J. M., Munt, S., et al. (2013) The first total synthesis of the cyclodepsipeptide pipecolidepsin A, Nat Commun 4, 2352.

[9] Liu, Y. Y., Wang, Y., Walsh, T. R., Yi, L. X., Zhang, R., Spencer, J., et al. (2016) Emergence of plasmid-mediated colistin resistance mechanism MCR-1 in animals and human beings in China: a microbiological and molecular biological study, Lancet Infect Dis 16, 161-168.

[10] Feng, Y. (2018) Transferability of MCR-1/2 Polymyxin Resistance: Complex Dissemination and Genetic Mechanism, ACS Infect Dis 4, 291-300.

[11] Li, Z., Chakraborty, P., de Vries, R. H., Song, C., Zhao, X., Roelfes, G., Scheffers, D. J., and Kuipers, O. P. (2020) Characterization of two relacidines belonging to a novel class of circular lipopeptides that act against Gram-negative bacterial pathogens, Environ Microbiol $22,5125-5136$.

[12] Mukherjee, S., and van der Donk, W. A. (2014) Mechanistic studies on the substrate-tolerant lanthipeptide synthetase ProcM, J Am Chem Soc 136, 10450-10459.

[13] Ge, J., Li, L., and Yao, S. Q. (2011) A self-immobilizing and fluorogenic unnatural amino acid that mimics phosphotyrosine, Chem Commun (Camb) 47, 10939-10941.

[14] Freire, F., Fisk, J. D., Peoples, A. J., Ivancic, M., Guzei, I. A., and Gellman, S. H. (2008) Diacid linkers that promote parallel beta-sheet secondary structure in water, J Am Chem Soc 130, 7839-7841.

[15] Chun, C., Lee, S. M., Kim, S. Y., Yang, H. K., and Song, S. C. (2009) Thermosensitive poly(organophosphazene)-paclitaxel conjugate gels for antitumor applications, Biomaterials 30, 2349-2360.

[16] Gude, M., Ryf, J., and White, P. D. (2002) An accurate method for the quantitation of Fmocderivatized solid phase supports, Letters in Peptide Science 9, 203-206.

[17] CLSI. Performance Standards for Antimicrobial Susceptibility Testing. 30th ed. CLSI supplement M100. Wayne, PA: Clinical Laboratory Standard Institute; 2020. 